\title{
Surface core-level shifts and atomic coordination at a stepped W(110) surface
}

\author{
D. M. Riffe, ${ }^{*}$ B. Kim, ${ }^{\dagger}$ and J. L. Erskine \\ Department of Physics, The University of Texas, Austin, Texas 78712
}

N. D. Shinn

Surface and Interface Science Department, Sandia National Laboratories, Albuquerque, New Mexico 87185-0344

(Received 11 April 1994; revised manuscript received 4 August 1994)

\begin{abstract}
Core-level $4 f_{7 / 2}$ photoemission spectra have been measured from a single, bifacial $\mathrm{W}$ crystal, which has both a flat $W(110)$ and a vicinal, stepped $W(110)[W(320)]$ surface. This procedure reduces uncertainties in the quantitative description of peaks in the spectra from $W(320)$. Various analyses, including nonlinear least-squares curve fitting, show that the average surface core-level shift (SCS) for W(320) is only $\sim-140 \mathrm{meV}$, compared to $-310 \mathrm{meV}$ for $\mathrm{W}(110)$ and that, at a maximum, only two of five terrace rows are isoelectronic to $\mathrm{W}(110)$ surface atoms. The absence of a large SCS for the step-edge atoms contradicts earlier interpretations of $\mathrm{W}(320)$ core-level spectra and departs significantly from expectations based on atomic-coordination models or tight-binding calculations of a bulk truncated surface. We suggest that systematic errors are responsible for the differences in reported core-level shifts for W(320). Implications of possible step-edge-driven atomic rearrangements are discussed.
\end{abstract}

\section{INTRODUCTION}

The physical and chemical properties of a solid surface depend critically upon the atomic configuration at the surface. For example, low-index densely packed surfaces such as bcc(110), fcc(111), or fcc(100) are often chemically quite distinct from their more open counterparts, bcc(111), bcc(100), and fcc(110). Atomically rough surfaces are expected to exhibit even greater chemical and electronic variety due to the presence of low-coordination surface atoms which can serve as nucleation or reaction sites. The thermodynamically stable vicinal surfaces (obtained by rotating the crystal a small angle from one low-index surface toward another) are ideal models for rough surfaces because low-coordination surface atoms may be systematically prepared and studied. These surfaces are commonly modeled as terraces of the nearby low-index plane separated by atomic steps, i.e., as a regularly spaced staircase of flat terraces each of which is structurally equivalent to the low-index surface from which it is derived. This accounts for the thermodynamic stability and the prevalence of vicinal surfaces on polycrystalline films and small particles that are used in chemical and materials applications.

A natural probe of atom-specific electronic properties of surfaces is core-level photoemission spectroscopy. From an electronic-structure viewpoint, differences in the core-level binding energies (BE's) between atoms in a metal principally reflect differences in local valencecharge density via the Coulombic interaction between the core and valence electrons. Experimental work on lowindex transition-metal surfaces has shown that core-level BE's are well correlated with the local atomiccoordination number, including second nearest neighbors, of a given atom., ${ }^{1,2}$ For tungsten, lower coordination results in lower binding energy. Hence the binding energy of a given tungsten core level will decrease in go- ing from bulk atoms to underlayer atoms to a densely packed surface and finally to an open surface. The decrease in binding energy is commonly referenced to the invariant core-level $\mathrm{BE}$ of bulk atoms, and is reported as a surface core-level shift (SCS). In the few cases where this simple atomic-coordination-BE relationship does not strictly hold, tight-binding electronic-structure calculations have been used to interpret the relative core-level shifts. ${ }^{1}$

If the terraces of a vicinal surface are isostructural to the corresponding low-index surface, core levels of the underlayer and top-layer interior-terrace atoms are expected to exhibit the same SCS's as the corresponding atoms on the low-index surface. Atoms near the step edge, due to different coordination numbers, are expected to have SCS's different from those atoms on the interior of the terrace. In particular, the step-edge atoms, which have the fewest nearest neighbors, are expected to have the largest SCS's. In studies of stepped Ir (Ref. 3) and Pt (Ref. 4) surfaces, this simple picture is consistent with the core-level data. However, since the intrinsic widths of the Ir and $\mathrm{Pt}$ core-level peaks are larger than their respective shifts, its is extremely difficult to make a definitive assignment of the SCS associated with each inequivalent atom. In earlier studies of stepped W surfaces, core-level spectra were well fit by assigning a discrete SCS to each inequivalent atom based upon its coordination number, with the largest SCS always being assigned to the step-edge atoms. ${ }^{5,6}$ These assignments have recently come under question since the intensity variations vs photon energy of the fitted core-level peaks in the earlier data are much greater than can be accounted for by final-state scattering effects. ${ }^{7}$

Here we report core-level photoemission measurements of the electronic structure of a vicinal W(110) surface, $\mathrm{W}(320)$. The $\mathrm{W}(320)$ surface is obtained by rotating the surface-normal vector $11.3^{\circ}$ from (110) toward the (100) 


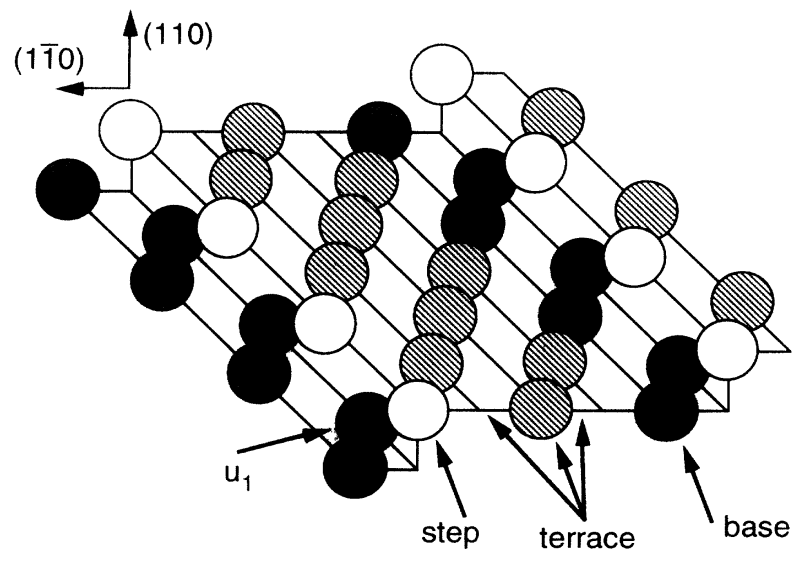

FIG. 1. Model of a bulk-truncated bcc(320) surface.

direction. The ideal (bulk-truncated) surface consists of (110) terraces five atoms wide separated by single atomic steps; see Fig. 1. Tungsten is an ideal material for such a study since the inherent width of the $4 f_{7 / 2}$ photopeaks is smaller than a typical SCS. Further, the interdiffusion of adsorbates is minimized since the surface energy of $W$ is so large, making $W$ an excellent template for the epitaxial growth of a wide variety of materials. ${ }^{8}$ In distinct contrast to the earlier reported work on $W(320),{ }^{5,6}$ we find that no lowest BE peak attributable to the edge atoms of the terrace exists. In fact, the overall surface spectral weight is much closer to the bulk than for the flat W(110) surface. Curve-fitting analysis indicates that, at a maximum, only two out of five terrace rows are electronically equivalent to $W(110)$. Our results show that earlier $\mathrm{W}(320)$ data $^{5,6}$ were misinterpreted due to systematic errors in binding-energy calibration and, more importantly, that coordination-number views and tight-binding calculations of bulk-truncated W(320) cannot explain the data. We suggest that the theoretical failures may be related to Smoluchowski smoothing of the charge density ${ }^{9}$ and/or possible relaxations or reconstructions of the surface layer.

\section{EXPERIMENTAL DETAILS}

The core-level data were obtained on the University of Texas/Sandia beam line on the vacuum ultraviolet (vuv) ring at the National Synchrotron Light Source at Brookhaven National Laboratory. Photon energies between 60 and $110 \mathrm{eV}$ were used to collect data at total resolutions between 120 and $140 \mathrm{meV}$.

The W crystal (1-cm diameter by $0.1 \mathrm{~cm}$ thick) was oriented (to within $\pm 0.5^{\circ}$ ), spark cut, and mechanically polished in order to simultaneously expose two different surface-crystallographic directions, the flat $W(110)$ and the stepped W(320) surface, each on one half of a single side of the crystal. The crystal was cleaned by standard oxidation-annealing techniques ${ }^{10}$ until core-level spectra of the W(110) portion of the surface exhibited no contamination features and could be well fit with parameters determined from previous studies of clean $\mathrm{W}(110){ }^{11}$ Low-energy electron diffraction (LEED) was used to veri- fy the periodicity of the vicinal surface.

The W(110) half of the sample provide not only an excellent check on surface cleanliness but also two other experimental controls. First, the W(110) surface spectra provide, due to their simplicity [one component from the first atomic layer (surface) and one from all other atoms (bulk)], an absolute energy reference for peaks in the W(320) spectra. ${ }^{12}$ Since the binding energy of the photoemission peak assignable to the bulk atoms is insensitive to surface orientation, we can accurately determine the binding energies of the W(320) surface peak and its components. Second, the W(110) spectra provide a first-order expectation of the overall surface/bulk intensity ratios from $\mathrm{W}(320)$ for any given photon energy and scattering geometry. In order to fully exploit these advantages, a typical run consists of flashing the sample to remove residual $\mathrm{H}$, recooling to $\sim 300 \mathrm{~K}$, and then measuring a $\mathbf{W}(320)$ spectrum. The sample is immediately reflashed, recooled, and translated parallel to the [001] direction in order to obtain a spectrum from the other surface with an identical scattering geometry. With this method matched pairs of W(320) and W(110) spectra were obtained.

\section{RESULTS AND ANALYSIS}

Four matched pairs of W(110) and W(320) spectra, obtained at a variety of photon energies and scattering geometries, are displayed in Fig. 2. In this and all other figures, spectra from W(110) are shown as the solid lines, while spectra from $W(320)$ are shown as filled circles. The following observations are characteristic of these and

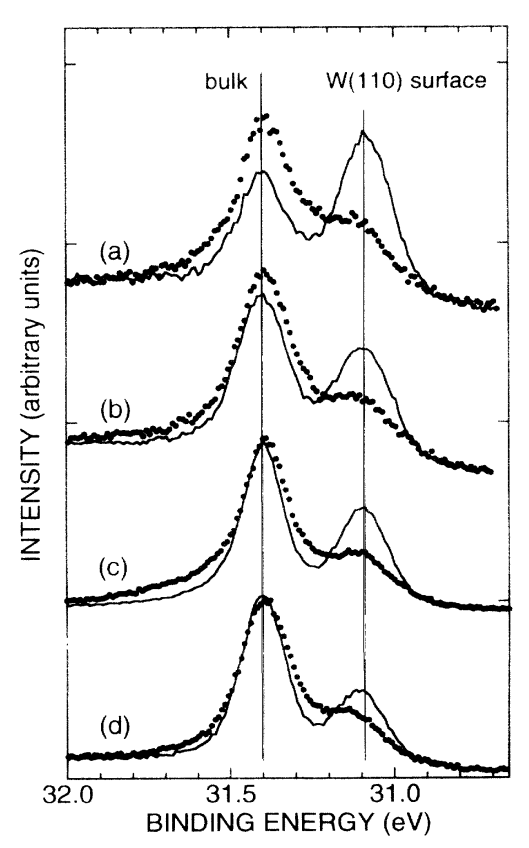

FIG. 2. Back-to-back data-set pairs taken on W(110) (solid lines) and W(320) (filled circles). The bulk and W(110) surfaceatom BE's are marked with the vertical lines. (a) and (b) Photon energy $=60 \mathrm{eV}$, two different scattering geometries. (c) Photon energy $=90 \mathrm{eV}$. (d) Photon energy $=65 \mathrm{eV}$. 
all other matched spectra which we have obtained. First, the strongest peak from $W(320)$ is always within \pm 20 meV of the W(110) bulk peak. Second, the spectral weight of the W(320) surface layer is spread over a wider energy range than on $W(110)$. Third, the $W(320)$ surface spectral weight is closer to the bulk BE than the surface spectral weight of $W(110)$, leading to a filling of the valley between the two peaks compared to $W(110)$ and an increase in the apparent bulk-peak height. In fact, some of the surface spectral weight has shifted so far that it appears on the high BE side of the W(110) bulk peak. This third observation contradicts results from previous studies, ${ }^{5,6}$ in which the step atoms were reported to induce an overall shift of surface spectral weight to comparatively lower BE away from the bulk peak.

In order better to appreciate the difference between our spectra and what is expected for a bulk-truncated staircase model of $\mathrm{W}(320)$, we have simulated a core-level spectrum of W(320) based on BE's for bulk-truncated W(320) which come from a tight-binding band-structure calculation of this surface. ${ }^{13,14}$ The simulated W(320) spectrum and its simulated W(110) counterpart are displayed in Fig. 3 as filled circles and the solid curve, respectively. As expected from the relative atomic coordination numbers, the step-atom component has the greatest binding-energy shift $(\mathrm{SCS}=-390 \mathrm{meV})$ and the underlayer component (SCS $=-126 \mathrm{meV}$ ) appears between the terrace (SCS $=-287 \mathrm{meV}$ ) and bulk components (SCS $\equiv 0 \mathrm{meV}$ ). The tight-binding calculations differ slightly from coordination-number expectations for the base atoms (SCS $=-333 \mathrm{meV}$ ). As seen in Fig. 1, the base atoms have higher coordination than the terrace atoms, so their SCS should be smaller. This SCS discrepancy for one row of base atoms has little effect on the overall position on the $W(320)$ surface spectral weight, however. Another insignificant difference is the slightly smaller SCS for the W(320) terrace atoms $(-287$ $\mathrm{meV}$ ) compared to that of the $\mathrm{W}(110)$ surface atoms $(-310 \mathrm{meV})$ in spite of equivalent coordinations.

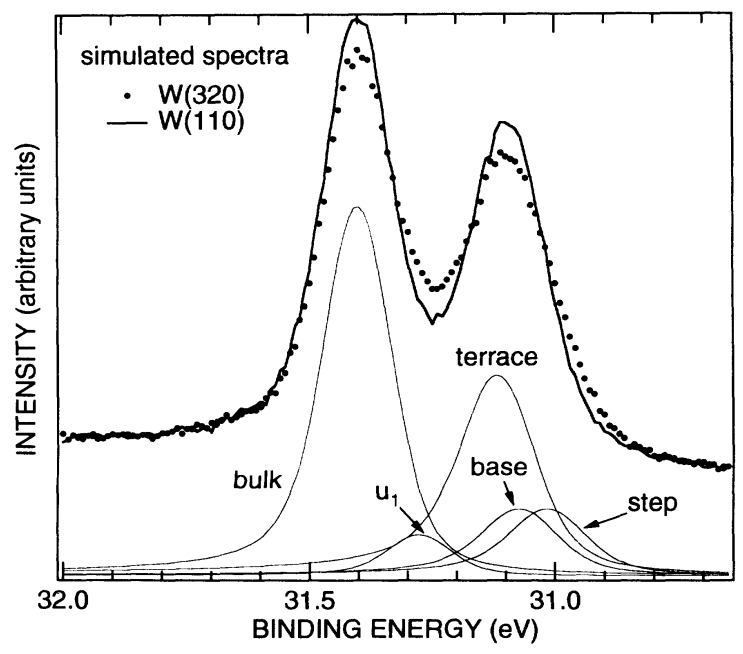

FIG. 3. Simulated core-level photoemission spectrum based on tight-binding calculations of Ref. 5. Also see Ref. 13.
Figure 3 clearly shows that the tight-binding calculations predict only subtle differences between $\mathrm{W}(110)$ and W(320) spectra. Even though the step-atom component is shifted $-103 \mathrm{meV}$ from the terrace atoms, a separate step-atom peak is not exhibited. The overall effect of the underlayer, base, and step-atom component shifts is a slight broadening of the surface peak with little change in its overall position.

A comparison of Figs. 2 and 3 shows that, although the bulk peak is the most intense feature in all cases, there exist major differences between our measured W(320) spectra and the stimulated one. First, and most obvious, the measured surface-peak peak intensity is considerably reduced compared to the simulated spectrum. This reduction implies that the binding energies of the surface atoms are spread over a wider energy range than the tight-binding theory predicts. The observation of the bulk-peak height in the measured W(320) spectra being, in general, larger than its $\mathrm{W}(110)$ counterpart is in contrast to the matched pair of simulated spectra. Clearly the tight-binding theory (of a bulk-terminated surface) fails to account for the observed core-level spectrum of the stepped W(320) surface.

A quantitative assessment of the average position of the surface spectral weight is made by calculating the $4 f_{7 / 2}$ binding-energy centriod $\langle\mathrm{BE}\rangle$ as a function of the fractional bulk signal. This allows us to compare spectra obtained at different collection geometries for which final-state scattering effects will change the surface:bulk intensity ratio. For all spectra we have defined $\langle\mathrm{BE}\rangle$ as $\left[\int d(\mathrm{BE})(\mathrm{BE})\left(4 f_{7 / 2}\right.\right.$ spectrum $\left.)\right] /\left[\int d(\mathrm{BE})\left(4 f_{7 / 2}\right.\right.$ spectrum)], where the integrations are done over a 1.4-eV window surrounding the $4 f_{7 / 2}$ peaks and the background has been subtracted from each spectrum before integration. The background was determined from simultaneous least-squares fitting of each spectrum and its background. The background depends only slightly on the choice of

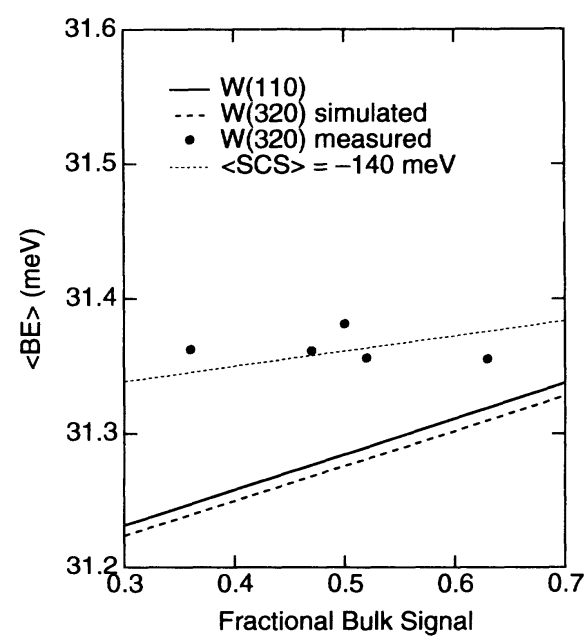

FIG. 4. Centroid ( $\langle B E\rangle)$ vs bulk fractional signal for theoretical and measured $W$ spectra. The solid line is from (simulated) W(110), the dashed line is from simulated $W(320)$, the filled circles are from measured $W(320)$ spectra, and the dotted line is for an average SCS of $-140 \mathrm{meV}$. 
model function used to describe the $4 f_{7 / 2}$ spectrum and thus has essentially no effect upon the value of $\langle B E\rangle$ presented in Fig. 3. The fractional bulk signal is defined as (integrated bulk $4 f_{7 / 2}$ intensity)/(total integrated $4 f_{7 / 2}$ intensity). For the measured data the fractional bulk signal was obtained from the matching W(110) spectra. In Fig. 4, the solid line is the $\langle B E\rangle$ for $W(110)$, for which there is only a single surface component at a $\mathrm{SCS}=-310 \mathrm{meV}$. The dashed line shows the result from the simulated $W(320)$ spectrum based on the tightbinding calculations shown in Fig. 3 and discussed above. The difference in $\langle\mathrm{BE}\rangle$ between $\mathrm{W}(110)$ and simulated $\mathrm{W}(320)$ is minimal regardless of the fractional bulk intensity. In contrast, the $\langle\mathrm{BE}\rangle$ for measured $\mathrm{W}(320)$ spectra over the same range, indicated by the solid circles in Fig. 4, are consistently at higher $\langle\mathrm{BE}\rangle$. This measured $\langle\mathrm{BE}\rangle$ for $\mathrm{W}(320)$ corresponds to an average SCS of only $\sim-140 \mathrm{meV}$ (dotted line in Fig. 4), a change of $+170 \mathrm{meV}$ compared to $\mathrm{W}(110)$.

\section{NONLINEAR \\ LEAST-SQUARES ANALYSIS}

Having shown that the tight-binding theory is inadequate for interpreting the $W(320)$ core-level spectra, we turn to least-squares analysis of the data. Our objective is to find a model (or models) based upon the least number of inequivalent tungsten atoms that provide statistically satisfactory fits to all of the data. As we show below, there are several model functions which satisfactorily describe the data; hence we cannot make a definitive assignment of individual $4 f_{7 / 2}$ BE's for each inequivalent atom represented in Fig. 1.

The data were nonlinear least-squares analyzed with a variety of models upon which were imposed the following physically motivated constraints. The lifetime width and singularity index of the bulk feature on W(320) were fixed to values obtained from earlier data on $W(110)(65 \mathrm{meV})$ and 0.035 , respectively). ${ }^{11}$ We found that the Gaussian width for the bulk peak, which is used to describe phonon broadening, instrumental resolution, and possible inhomogeneous effects, was never significantly different from that of the matched W(110) spectrum. For other components, which account for the various surface atoms [and possibly the underlayer row $u_{1}$ (Ref. 15)], the lifetime width and singularity index were constrained to

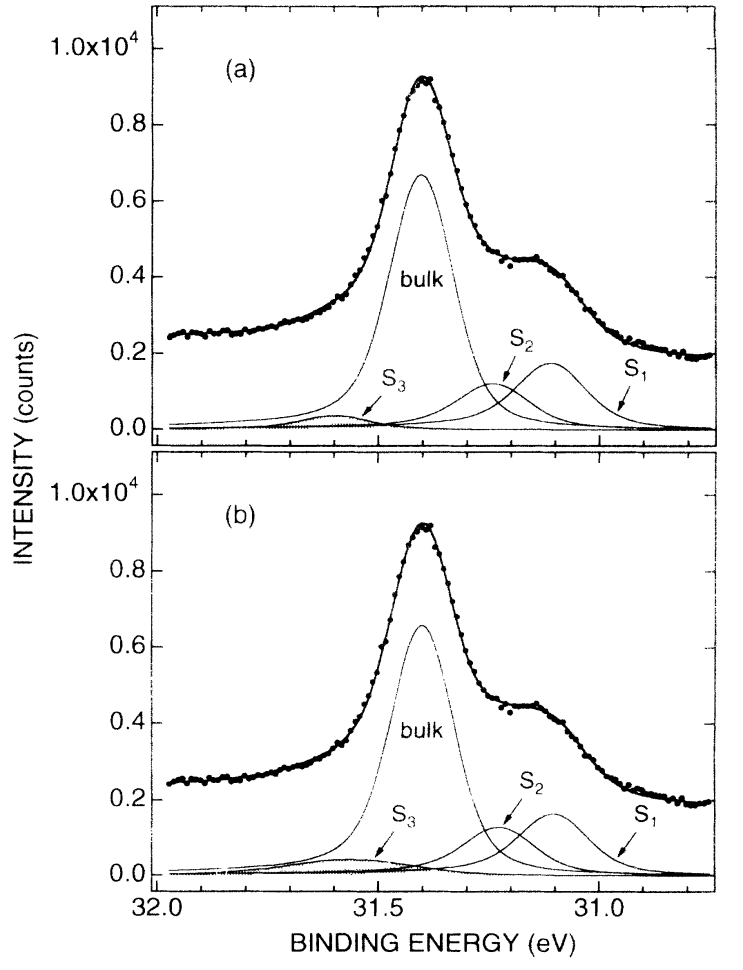

FIG. 5. Four-component analysis of $\mathrm{W}(320)$ spectrum. In (a) all surface components have the same shape. In (b) $S_{3}$ has a Gaussian width twice as large as the other surface components.

$\mathrm{W}(110)$ surface-atom values $(80 \mathrm{meV}$ and 0.063 , respectively). Since these line-shape parameters are not greatly different from those for bulk $\mathbf{W}$, it is expected that they should not change appreciably between the surface of W(320) and W(110). Details of the surface Gaussian width varied from model function to model function and are described as necessary below. The underlying background intensity was modeled with either a linear or power-law function. In order to ensure convergence and consistency of the fitting parameters we found it necessary to simultaneously fit pairs of W(320) spectra.

Using these constraints we find that equally acceptable descriptions of $\mathbf{W}(320)$ spectra can be obtained with either three or four separate components. Fits with more than four components were unsuccessful because they generally resulted in two components with nearly identi-

TABLE I. Summary of least-squares analysis with model functions illustrated in Figs. 5 and 6.

\begin{tabular}{|c|c|c|c|c|c|c|c|}
\hline \multirow[b]{3}{*}{ Figure } & \multirow{3}{*}{$\begin{array}{c}\frac{I\left(B_{320}\right)-I\left(B_{110}\right)}{I\left(B_{110}\right)} \\
( \pm 5 \%)\end{array}$} & \multicolumn{6}{|c|}{ Surface components } \\
\hline & & \multicolumn{2}{|c|}{$S_{1}$} & \multicolumn{2}{|c|}{$S_{2}$} & \multicolumn{2}{|c|}{$S_{3}$} \\
\hline & & $\mathbf{S C S}^{\mathrm{a}}$ & $I^{\mathrm{b}}$ & SCS & $I$ & SCS & $I$ \\
\hline $5(a)$ & $+6 \%$ & -310 & $50 \pm 3$ & -200 & $36 \pm 6$ & +190 & $13 \pm 3$ \\
\hline $5(b)$ & $+4 \%$ & -315 & $43 \pm 5$ & -180 & $34 \pm 5$ & +140 & $21 \pm 4$ \\
\hline $6(a)$ & 0 & -275 & $64 \pm 6$ & +50 & $36 \pm 6$ & & \\
\hline $6(b)$ & $+5 \%$ & -270 & $60 \pm 5$ & -80 & $40 \pm 5$ & & \\
\hline
\end{tabular}

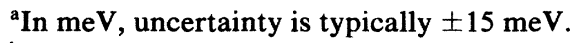

b\% of surface spectral weight. 
cal binding energies, and consistency between data sets could not be achieved.

Figure 5(a) displays the results (for one data set) of the most conventional model function used for analyzing the $\mathrm{W}(320)$ surface. The components in this fit are constrained to have the same Gaussian width as their W(110) counterparts. With this requirement four components are found to be necessary for adequate representation of the data. The results are summarized in Table I. In favor of this model function is an $S_{1}$ SCS identical to that of $W(110)$ surface atoms. Also, the bulk-peak intensity is nearly identical to that from the flat surface. However, the division of surface spectral weight $I\left(S_{i}\right)$ among the three surface features is somewhat problematic. Since there are five atomic rows of surface atoms per terrace, each row should ideally produce $20 \%$ of the surface spectral weight. Therefore $I\left(S_{3}\right)$ only accounts for slightly more than $\frac{1}{2}$ of a terrace row.

In order to increase the $S_{3}$ intensity the restriction on the Gaussian width was lifted. Figure 5(b) shows a fit to one data set where the Gaussian width of $S_{3}$ is set to twice the value of the other surface lines. The intensity of $S_{3}$ now corresponds to one row of terrace atoms. Intensities $I\left(S_{1}\right)$ and $I\left(S_{2}\right)$ each account for two rows per terrace. With this description the bulk intensity is again slightly larger than for the flat surface.

While the model displayed in Fig. 5(b) is realistic from the viewpoint of component intensities, we find that three components are also sufficient for describing the data, although the constraints on the surface Gaussian widths must be lifted. One such fit is illustrated in Fig. 6(a).

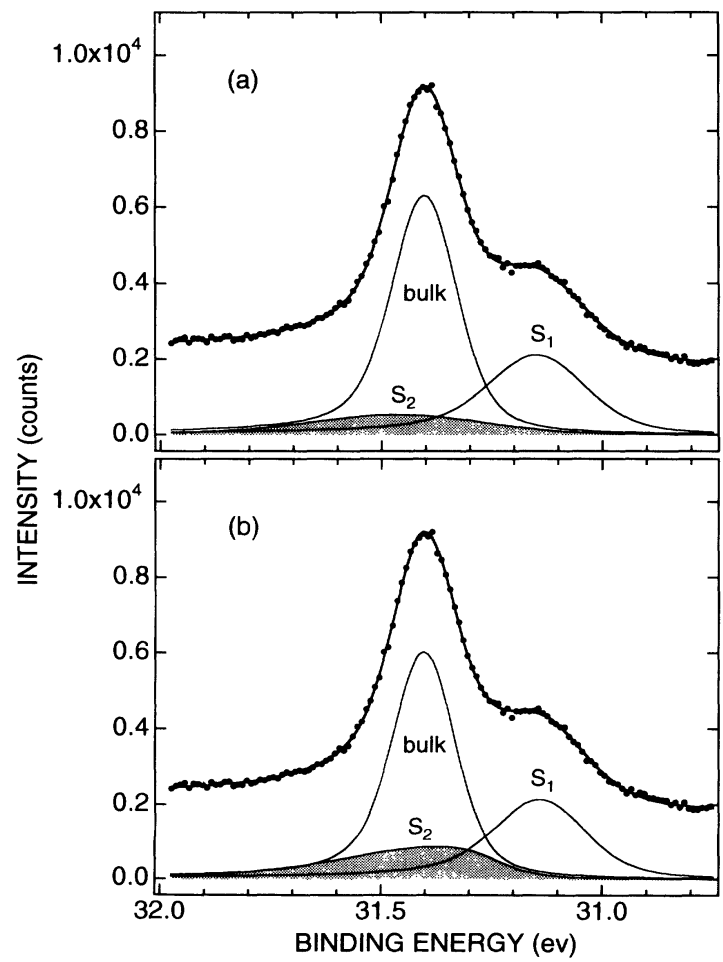

FIG. 6. Three-component analysis of the W(320) spectrum. In (a) all components have a symmetric Gaussian component. In (b) $S_{2}$ has asymmetric Gaussian character.
With this model we found it necessary to constrain the position of $S_{2}$. Otherwise it grows in intensity at the expense of the bulk component, a clearly unphysical result. A slight variation on this model function is illustrated in Fig. 6(b), in which $S_{2}$ is given asymmetric Gaussian character. Here the only additional constraint is that the low-BE Gaussian width of $S_{2}$ is set equal to the surface Gaussian width on W(110). Without this control the low-BE Gaussian width of $S_{2}$ unphysically narrows below that for the flat surface. Again, the general results of both three-line analyses are included in Table I. The resultant surface intensities in either three-line model function implies that $S_{1}$ accounts for three rows per terrace, and $S_{2}$ accounts for the remaining two rows.

\section{DISCUSSION}

Our measurements are in marked disagreement with both the tight-binding theory ${ }^{5}$ and coordination-number concepts of a bulk-terminated W(320) surface and with earlier experimental data. ${ }^{5,6}$ As summarized in Table II, the coordination-number approach predicts the step atoms to have a SCS $<-310 \mathrm{meV}$, while the tightbinding theory predicts $-390 \mathrm{meV}$. The previous experimental results are -590 and $-420 \mathrm{meV}$. In contrast, our curve-fitting analysis shows that all surface-related components have SCS's somewhere between -310 and $+200 \mathrm{meV}$. Additionally, the $\langle\mathrm{BE}\rangle$ analysis (Fig. 4) shows that the average SCS for W(320) is $\sim-140 \mathrm{meV}$ compared to $<-310 \mathrm{meV}$ for both theories and the earlier experimental data. We now discuss the discrepancies with theory and then show that a systematic BE shift in each of the earlier data sets reconciles all three experimental studies.

We first consider the possibility that the surface is isostructural to $\mathrm{W}(110)$; i.e., it is bulk terminated with no relaxation or reconstruction of the surface atoms. In this case it is clear that both theoretical approaches must be discarded as flawed. One physical process which may help to explain the failure of coordination-number ideas is smoothing of the electron density at step edges, i.e., the Smoluchowski effect. ${ }^{9}$ Such smoothing will alter the charge density of the step atoms from an atomic description upon which coordination-number ideas are predicated. This viewpoint is supported by recent electronicstructure calculations of a stepped $\mathrm{Al}(331)$ surface ${ }^{16}$ where one would naively expect directional bonding to favor an atomic-coordination approach. Instead, the electron density at steps was found to be better represented by microfacets of low-index surfaces rather than discrete atoms with missing neighbors.

The possibility that the surface is not bulk terminated comes from considering the accuracy of tight-binding cal-

TABLE II. Comparison of SCS values for step-edge atoms on $\mathrm{W}(320)$ in $\mathrm{meV}$.

\begin{tabular}{ccccc}
\hline \hline This work & $\begin{array}{c}\text { Coordination- } \\
\text { number } \\
\text { approach }\end{array}$ & $\begin{array}{c}\text { Tight- } \\
\text { binding } \\
\text { theory }\end{array}$ & Ref. 5 & Ref. 6 \\
\hline$>-310$ and $<+200$ & $<-310$ & -390 & -590 & -420 \\
\hline
\end{tabular}


culations of low-index $\mathrm{W}$ and Ta surfaces which have successfully predicted the relative SCS's on these associated surfaces. ${ }^{1}$ There are several physical reasons why the stepped W(320) surface might be relaxed and/or reconstructed even though its flat counterpart $\mathbf{W}(110)$ is not. First, the existence of the step edge itself with any local Smoluchowski smoothing of the charge density away from a discrete-atom missing-neighbor picture could drive the step-edge (and possibly other) atoms away from their bulk-truncated positions. In a local chemicalbonding picture this corresponds to the step atoms attempting to saturate their bonds by changing their positions. This could be achieved either by relaxation toward the bulk or through some sort of reconstruction parallel to the surface. How localized (to the step edge) such a rearrangement might be is hard to suggest with any confidence, since the bonding nature of solid $\mathrm{W}$ is somewhere between covalent and metallic due to the partially localized nature of the $5 d$ electrons which largely comprise the valence band. We thus consider both steplocalized and global rearrangements in discussing the data.

If a localized bonding picture is correct, then any reconstruction or relaxation driven by the step edges should leave at least some of the interior terrace atoms isoelectronic to $\mathbf{W}(110)$ surface atoms. For this possibility the least-squares analysis shows that at most only two atomic rows remain unperturbed $\left(S_{1}\right.$ in Fig. 5). ${ }^{17}$ The step atom and its nearest neighbors (the base atom and nearest terrace atom) would then be associated with the surface spectral weight which sits close to the bulk feature. We note that any such localized arrangement must be consistent with our LEED observations that the local (length scale smaller than a terrace width) periodicity on the stepped surface remains the same as on W(110). However, a disordered reconstruction, where the disorder is driven by temperature, would not be readily apparent in our observations.

There is also the possibility that a more global rearrangement occurs on this surface. On $W(110)$ a $p(1 \times 1)$ reconstruction (which preserves the unit-cell size) has been observed upon $\mathrm{H}$ coverage in excess of 0.5 monolayer. ${ }^{18}$ It was suggested that the $\mathrm{H}$ sits in isodirectional threefold sites and thus drives a lateral shift of the surface layer along the $(1 \overline{1} 0)$ direction. Since $(1 \overline{1} 0)$ is perpendicular to the step edges on W(320), the presence of the steps themselves may provide an asymmetry sufficient to induce a similar reconstruction. For such a reconstruction all of the surface atoms would likely experience similar core-level shifts toward higher binding energy (away from the bulk-truncation expectation) due to an effective increase in coordination. We obtain a numerical estimate of such a reconstruction-induced core-level shift on W(320) from a previous core-level study of the $\mathrm{H} / \mathrm{W}(110)$ system. $^{19}$ In that study an increase in the surface-atom core-level binding energy of $130 \mathrm{meV}$ due solely to the reconstruction (aside from a chemical shift due to direct bonding with $\mathbf{H}$ ) was inferred. This is remarkably close to the $170-\mathrm{meV}\langle\mathrm{BE}\rangle$ increase between $\mathbf{W}(320)$ and $\mathbf{W}(110)$. Although a local atomiccoordination picture still fails in this scenario for $\mathbf{W}(320)$,

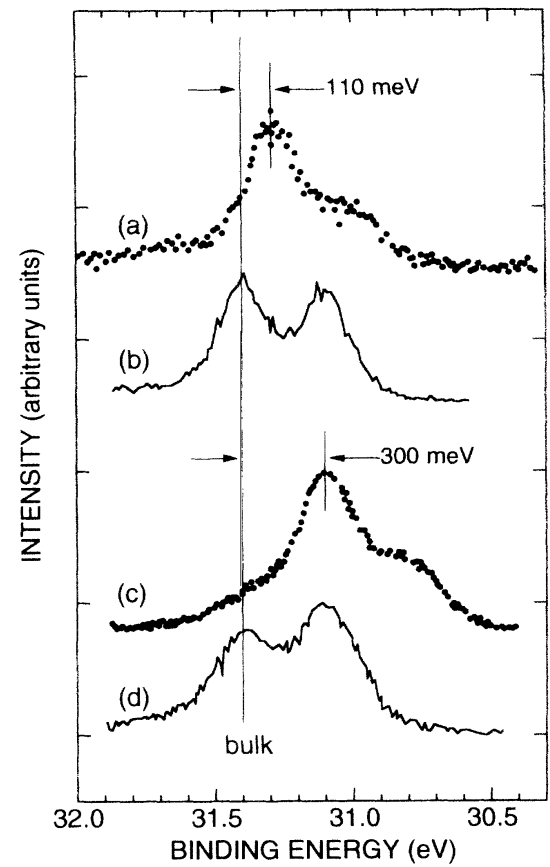

FIG. 7. Core-level photoemission data of $W(320)$ and $W(110)$ from Refs. 5 and 6. Filled circles are from $W(320)$ and solid lines from $\mathbf{W}(110)$. Curves $a$ and $b$ are from Ref. 6. Curves $c$ and $d$ from Ref. 5 .

due to the large observed spread in surface spectral weight, a global reconstruction such as this where all of the atoms are similarly displaced agrees on average with atomic-coordination estimates.

We now offer an explanation for the difference between our study and earlier core-level studies, where it was concluded that the step atoms produce a low-BE feature consistent with an atomic-coordination or tight-binding interpretation of a bulk-truncation model of W(320), ${ }^{5,6}$ In Fig. 7 we display pairs of $W(110)$ and $W(320)$ data from

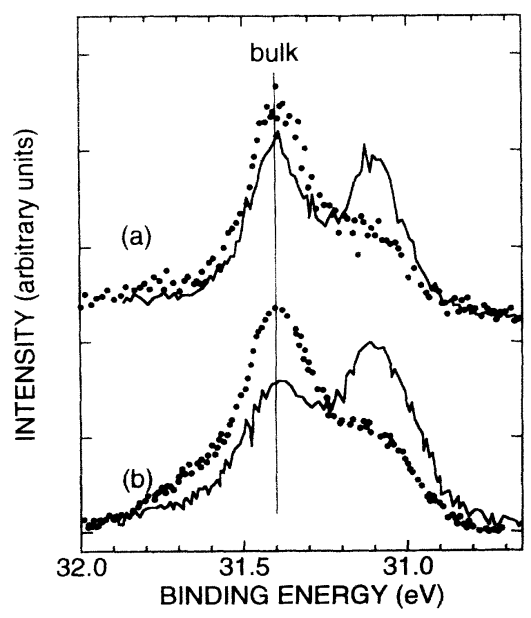

FIG. 8. Same data as in Fig. 7 except $W(320)$ spectra have been shifted up in BE by $110 \mathrm{meV}$ (a) (Ref. 6) and $300 \mathrm{meV}$ (b) (Ref. 5). 
these two previous studies. Curves $a$ and $b$ in Fig. 7 are $\mathbf{W}(320)$ and $\mathbf{W}(110)$ spectra, respectively, from Ref. 6, while curves $c$ and $d$ are analogous spectra from Ref. 5. In both sets of data it does indeed appear that a low-BE feature attributable to the step atoms exists. However, in comparison with the present data with accurate relative BE's of W(320) and W(110), it seems that in both previous cases the W(320) spectra were inappropriately shifted to lower BE. Judging from the position of the most intense feature in our W(320) data, which is within $20 \mathrm{meV}$ of the bulk BE, the W(320) spectra in Ref. 6 have been shifted by $\sim-110 \mathrm{meV}$, while the W(320) spectra in Ref. 5 have been shifted by $\sim-300 \mathrm{meV}$. In Fig. 8 we again display the previous spectra but have shifted the W(320) spectra upwards in BE by these amounts, $110 \mathrm{meV}$ for the data from Ref. 6 and $300 \mathrm{meV}$ from Ref. 5. With these BE corrections, the previously reported data are now strikingly similar to each other and to our own data of Fig. 2.

While it is difficult to pinpoint the sources of bindingenergy error in the earlier investigations, we offer the following suggestions. It appears that in Ref. 5 the W(320) bulk peak was incorrectly taken for one due to terrace atoms. In Ref. 6 the error apparently arose in the fitting process in which a peak located above the bulk-peak position was misidentified as the bulk peak. We note that the authors of Ref. 6 quote an uncertainty of $\pm 50 \mathrm{meV}$ in their bulk-peak position, a value not much smaller than the suggested error of $110 \mathrm{meV}$.

In summary, we have presented core-level photoemission data from $W(320)$ which are in distinct contrast to microscopic calculations and coordination-number expectations for this surface. We have discussed the possibility that a different theoretical approach may be needed to understand stepped-surface SCS's and have considered possible reconstructions which may affect the SCS's. We have further reconciled two earlier sets of data by invoking systematic BE errors in the previous studies. Our results raise the question of the applicability of coordination-number interpretations of core-level spectra from other high-index surfaces and highlight the need for more first-principles calculations of core-level binding energies of stepped-surface systems.

\section{ACKNOWLEDGMENTS}

This work was supported by NSF Contract No. DMR93-03091 and the Robert A. Welch Foundation (University of Texas) and by the DOE division of Material Sciences under Contract No. DE-ACO4-94AL85000 (Sandia National Laboratories). The National Synchrotron Light Source is supported by the DOE Division of Material Science and Division of Chemical Sciences.
*Present address: Dept. of Physics, Utah State University, Logan, UT 84322.

†Present address: P. L. S., Pohang University of Science and Technology, Republic of Korea.

${ }^{1} D$. Spanjaard, C. Guillot, M. C. Desjonqueres, G. Treglia, and L. Lecante, Surf. Sci. Rep. 5, 1 (1985).

${ }^{2}$ A. Rosengren, Phys. Rev. B 24, 7393 (1981).

3J. F. van der Veen, D. E. Eastman, A. M. Bradshaw, and S. Holloway, Solid State Commun. 39, 1301 (1981).

${ }^{4}$ G. Apai, R. C. Baetzold, P. J. Jupiter, A. J. Viescas, and I. Lindau, Surf. Sci. 134, 122 (1983).

${ }^{5}$ D. Chaveau, P. Roubin, C. Guillot, J. Lecante, G. Tréglia, M. C. Desjonqueres, and D. Spanjaard, Solid State Commun. 52, 635 (1984)

${ }^{6}$ K. G. Prucell, J. Jupille, and D. A. King, Surf. Sci. 208, 245 (1989).

${ }^{7}$ B. Kim, J. Chen, J. L. Erskine, W. N. Mei, and C. M. Wei, Phys. Rev. B 48, 4735 (1993).

${ }^{8}$ N. D. Shinn, B. Kim, A. B. Andrews, J. L. Erskine, K. J. Kim, and T. H. Kang, in Applications of Synchrotron Radiation Techniques to Materials Science, edited by D. L. Perry, N. D. Shinn, R. L. Stockbauer, K. L. D'Amico, and L. J. Terminello, MRS Symposia Proceedings No. 307 (Materials Research Society, Pittsburgh, 1993), p. 167.

${ }^{9}$ R. Smoluchowski, Phys. Rev. 60, 661 (1941).

${ }^{10}$ R. G. Musket, W. McLean, C. A. Colmenares, D. M. Makowiecki, and W. J. Siekhaus, Appl. Surf. Sci. 10, 143 (1982).

${ }^{11}$ D. M. Riffe, G. K. Wertheim, and P. H. Citrin, Phys. Rev. Lett. 63, 1976 (1989).

${ }^{12}$ We use "peak" to denote a spectral feature, and "component" to denote the contributions (to a peak or spectrum) from ine- quivalent tungsten atoms.

${ }^{13}$ The tight-binding calculations predict SCS's for W(320) as follows: $u_{1}$ : $-110 \mathrm{meV}$; terrace: $-250 \mathrm{meV}$; base: $-290 \mathrm{meV}$; and step: $-340 \mathrm{meV}$ (see Ref. 5). For W(110) the tightbinding calculations predict a SCS of $-270 \mathrm{meV}$ for the surface atoms [M. C. Desjonqueres, D. Spanjaard, Y. Lassailly, and C. Guillot, Solid State Commun. 34, 807 (1980)]. In Fig. 3 , the discussion in the text, and Table II, we have thus scaled the theoretical predictions by the experimental/theoretical ratio of $310 / 270$ for the SCS of the W(110) surface atoms.

${ }^{14}$ The number of counts in the bulk peak of the $\mathrm{W}(110)$ simulated spectrum was set to 6000 , typical of data in the experiment. Gaussian-distributed counting noise was added to the simulated data. In both simulated spectra $[\mathrm{W}(110)$ and $\mathrm{W}(320)]$ the bulk/surface integrated-intensity ratio was fixed at $1 / 1$, in the middle of the range of the measured data. The individual intensities of the peaks in the $W(320)$ spectrum are from a simple escape-depth model, i.e., differences in finalstate diffraction between $\mathrm{W}(110)$ and $\mathrm{W}(320)$ have been ignored. The similarity of the differences between measured $W(320)$ and $W(110)$ spectra shows that the effects of photoelectron diffraction are largely negligible when comparing matched (320) and (110) data sets. Hence the approximation of ignoring differences in diffraction between matched data is reasonably valid.

${ }^{15}$ In the discussion which follows we have decided not to explicitly discuss the $u_{1}$ layer. Given the expected small contribution to the overall spectral weight $(\sim 5 \%)$, closeness to the bulk line, and uncertainties in other spectral weights $( \pm \sim 5 \%)$, it is unreasonable to expect that one can identify a separate peak due to these atoms.

16J. S. Nelson and P. J. Feibelman, Phys. Rev. Lett. 68, 2188 
(1992).

${ }^{17}$ While in the three-component fits $S_{1}$ accounts for three rows, its large Gaussian width indicates that the rows which comprise this component are not all themselves isoelectronic, and hence at most two of the rows are equivalent to $\mathrm{W}(110)$ surface atoms.
${ }^{18}$ J. W. Chung, S. C. Ying, and P. J. Estrup, Phys. Rev. Lett. 56, 749 (1986); M. Altman, J. W. Chung, P. J. Estrup, J. M. Kosterlitz, J. Prybyla, D. Sahu, and S. C. Ying, J. Vac. Sci. Technol. A 5, 1054 (1987).

${ }^{19}$ D. M. Riffe, G. K. Wertheim, and P. H. Citrin, Phys. Rev. Lett. 65, 219 (1990). 


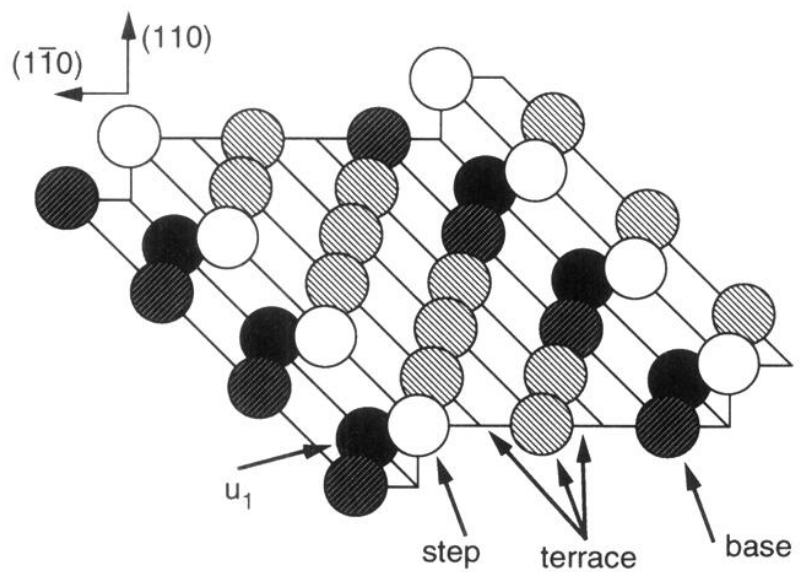

FIG. 1. Model of a bulk-truncated bcc(320) surface. 